extrapyramidal and neurocognitive symptoms have now resolved suggesting that this extrapyramidal syndrome is reversible.

Conclusion Rifaxamin is efficacious in the treatment of the Parkinsonian phenotype of HE in this small case series which appears to be independent of ammonia lowering. Larger clinical trials are now warranted.

Competing interests None declared.

\section{PTU-021 DO ALL REGENERATIVE NODULES BECOME HEPATOCELLULAR CARCINOMA? THE OUTCOME OF 4 YEARS MRI SURVEILLANCE}

doi:10.1136/gutjnl-2012-302514c.21

${ }^{1} \mathrm{~B}$ Hogan, ${ }^{*}{ }^{1} \mathrm{P} \mathrm{M}$ Trembling, ${ }^{1} \mathrm{~S}$ Tanwar, ${ }^{2} \mathrm{D}$ Yu, ' J P O'Beirne, ${ }^{1} \mathrm{~W} M$ Rosenberg. ${ }^{1}$ Centre for Hepatology, University College London, London, UK; ${ }^{2}$ Department of Radiology, Royal Free Hospital, London, UK

Introduction There are few epidemiological data on the longitudinal follow-up of nodular lesions in cirrhotic patients.

Methods The Royal Free Hospital database was searched for all reports of MRI scans of the liver in which the term "nodule" was used. 630 such scans were identified in 369 individual patients between 1 January 2006 and 1 January 2011. Patients were then excluded if there was <1-year follow-up (45), if hepatocellular carcinoma (HCC) was identified on the initial scan (135), if an alternative diagnosis was made (129) or if no significant arterialised lesion was reported despite previous suspicion on ultrasound scanning (31). Retrospective analysis was, therefore, performed on 29 cirrhotic patients with a diagnosis of regenerative, indeterminate or dysplastic arterialised nodules at baseline and $>1$ year total followup with MRI and alpha-fetoprotein (AFP) surveillance.

Results The median age at first scan was 55 years (range 36-73) and the most common aetiologies of cirrhosis were hepatitis C $(55 \%)$, hepatitis B (10\%) and alcohol (25\%) with $10 \%$ other. The median follow-up period was 22 months (12-56) and the median number of scans $4(2-10)$. At baseline nodules were described as indeterminate in $48 \%$, regenerative in $45 \%$ and dysplastic in $7 \%$. The prevalence of HCC after 1, 2, 3 and 4 years of follow-up was 5 (17\%), 9 (31\%), 11 $(38 \%)$ and $11(38 \%)$ respectively, with the highest incidence occurring in the first 2 years of follow-up ( $82 \%$ of cases). The median size of nodule at baseline in those who developed HCC was $10 \mathrm{~mm}$ (5-18) and it was $9 \mathrm{~mm}(5-26)$ in those who did not. There was no association between the description of the nodule and the likelihood of developing HCC (five arising from indeterminate and 6 from regenerative nodules) and the two nodules initially described as dysplastic did not transform to tumour. AFP results were not informative.

Conclusion $31 \%$ of lesions described as arterialised nodules when first scanned developed into HCC within 2 years and initial radiological features consistent with "regenerative" nodules are not reassuring. Patients with discrete arterialised nodules should be enrolled in enhanced surveillance programmes.

Competing interests None declared.

\section{PTU-022 PREOPERATIVE NEUTROPHIL: LYMPHOCYTE RATIO DOES NOT PREDICT POST-OPERATIVE COMPLICATIONS FOLLOWING HEPATIC RESECTION}

doi:10.1136/gutjnl-2012-302514c.22

C E Western, ${ }^{*}$ S Aroori, M Bowles, D Stell. Hepatopancreatobiliary, Derriford Hospital, Plymouth, UK

Introduction Neutrophil:lymphocyte ratio (NLR) is a marker of systemic inflammation and, if high, has been shown in several studies to be correlated with adverse outcome following operative intervention in many conditions. ${ }^{1-3}$ We attempted to test this association by analysing pre-operative NLR in all patients undergoing liver resection in our unit and attempting correlation with markers of adverse outcome in the form of post-operative complications and length of inpatient stay.

Methods Our unit is a regional tertiary referral centre for hepatic surgery undertaken for both benign and malignant disease. A database of patient demographics, radiological and histological findings and blood tests for this cohort has been prospectively maintained since 2005 and the data has been retrospectively analysed for this study. A NLR of $>5$ was considered elevated.

Results Between 15 July 2005 and 27 September 2011, 377 hepatic resections were performed. $62 \%$ were for colorectal carcinoma metastases, $6 \%$ for other metastases, $8 \%$ for benign disease, $7 \%$ for cholangiocarcinoma, $7 \%$ for hepatocellular carcinoma, $4 \%$ for gallbladder cancer and $2 \%$ for neuroendocrine tumours. Median patient age was 65 and $56 \%$ were male. Our median NLR was 2.5. Overall morbidity was $26 \%$. Post-operative complications seen include bile leak $8 \%$, liver failure $1 \%$ and post-operative death $2 \%$. There were no intra-operative deaths. Intra-operatively, $19 \%$ of patients required an average of 3.8 units packed cell transfusion, $6 \%$ an average of 3.7 units FFP and 2\% 1.5 pool platelets. When NLR was correlated against post-operative complications, there was seen to be no association ( $p>0.5$ ). Median length of inpatient stay was 8 days (range 3 to 70 days). There was also no correlation demonstrable between NLR and length of inpatient stay ( $p=0.23$ ).

Conclusion Preoperative NLR does not appear to be a useful predictor of post-operative outcomes in the form of complications and post-operative hospital stay in patients undergoing liver resection.

\section{Abstract PTU-022 Table 1}

\begin{tabular}{lc}
\hline Surgical procedure & Percentage \\
\hline Right hemihepatectomy & $29 \%$ \\
Left hemihepatectomy & $12 \%$ \\
(Partially) extended right hemihepatectomy & $13 \%$ \\
(Partially) extended left hemihepatectomy & $6 \%$ \\
Left lateral excision & $9 \%$ \\
Wedge resection & $24 \%$ \\
Excision gallbladder bed & $4 \%$ \\
Other & $3 \%$ \\
\hline
\end{tabular}

Competing interests None declared.

\section{REFERENCES}

1. Bhutta H, Agha R, Wong J, et al. Neutrophil-lymphocyte ratio predicts medium-term survival following elective major vascular surgery: a cross-sectional study. Vasc Endovascular Surg 2011;45:227-31

2. Gomez D, Farid S, Malik HZ, et al. Preoperative neutrophil-to-lymphocyte ratio as a prognostic predictor after curative resection for hepatocellular carcinoma. World $\mathrm{J}$ Surg 2008; 32:1757-62.

3. Jung MR, Park YK, Jeong 0, et al. Elevated preoperative neutrophil to lymphocyte ratio predicts poor survival following resection in late stage gastric cancer. J Surg Oncol 2011;104:504-10.

\section{PTU-023 LONG-TERM OUTCOMES FOLLOWING DRUG-ELUTING BEAD TRANSARTERIAL CHEMOEMBOLISATION (DEB-TACE) AS PART OF MULTIMODALITY TREATMENT FOR HEPATOCELLULAR CARCINOMA}

doi:10.1136/gutjnl-2012-302514c.23

${ }^{1} \mathrm{C}$ Lever, ${ }^{*} \mathrm{P}$ Kumar, ${ }^{3} \mathrm{~S}$ George, ${ }^{2} \mathrm{~N}$ Hacking, ${ }^{2} \mathrm{~B}$ Stedman, ${ }^{2} \mathrm{D}$ Breen, ${ }^{4} \mathrm{~N}$ Pearce, ${ }^{1} \mathrm{M}$ Wright. ${ }^{1}$ Department of Hepatology, Southampton University Hospital Trust, 
Southampton, UK; ${ }^{2}$ Department of Radiology, Southampton University Hospital Trust, Southampton, UK; ${ }^{3}$ Department of Public Health, Southampton University Hospital Trust, Southampton, UK; ${ }^{4} H P B$ Surgery, Southampton University Hospital Trust, Southampton, UK

Introduction Treatment protocols for hepatocellular carcinoma (HCC) are evolving rapidly. We sought to determine long-term outcomes in patients with HCC treated with DEB-TACE as stand alone or part of multimodality treatment at a single centre.

Methods Our departmental database of HCC, diagnosed using EASL radiological criteria, was reviewed retrospectively. From August 2006 to January 2011, 80 patients (60 males/20 females) underwent DEBTACE some of which also had surgery and/or percutaneous ablation. A total of 186 episodes of DEB-TACE treatment were performed (minimum 1 episode and maximum 8 episodes per patient). $37.5 \%$ of patients presented with multifocal disease, a further $7.5 \%$ presented with tumour plus satellites. The mean MELD (model for end-stage liver disease) in this cohort was 9.53 (range 6-22). Demographics, procedural details, clinical measures and outcomes were studied. Median age was 69 years (range 33-87). All patients were included in the survival analysis. Overall survival was described using Kaplan-Meier methods.

Results 53 patients with a median tumour size of $49 \mathrm{~mm}$ (range $12-163 \mathrm{~mm}$ ) were treated with DEB-TACE alone with a mean number of procedures of 2.1. Here median survival was 28.5 months (798 days). The 1 - and 3-year survival rates in this group where $66 \%$ and $38 \%$ respectively. 27 patients (median tumour size of $40 \mathrm{~mm}$ (range 12-100 $\mathrm{mm}$ )) were treated with a combination of therapies and at 55 months (1540 days) the median survival had not been reached. Survival in this group was $51.1 \%$ at time data collection. In this group the 1- and 3-year survival rates were $86 \%$ and $64 \%$ respectively. In our cohort of patients DEB-TACE both with or without combination therapies, resulted in median survival of 44 months. Overall survival rates at 1 - and 3 -years were $74.5 \%$ and $50.3 \%$ respectively. There were no deaths at 30 days following a DEB-TACE episode.

Conclusion In our centre this procedure is safe and well tolerated with multimodality treatment showing an improved survival outcome. Our results highlight the importance of a multidisciplinary approach with the application of multimodal therapy in the management of HCC with an improved survival for appropriately selected patients.

Competing interests None declared.

\section{PTU-024 A "REAL WORLD" CONTROLLED STUDY OF LIVER STIFFNESS MEASURED BY ARFI (ACOUSTIC RADIATION FORCE IMPULSE) ELASTOGRAPHY IN HOSPITALISED PATIENTS WITH DECOMPENSATED ALCOHOLIC LIVER DISEASE (ALD): A NEW PARADIGM IN ASSESSMENT OF SEVERITY AND PROGNOSIS?}

doi:10.1136/gutjnl-2012-302514c.24

${ }^{1} \mathrm{D}$ I Sherman, ${ }^{*}{ }^{1} \mathrm{~K}$ Kandiah, ${ }^{2} \mathrm{M}$ Jagtiani, ${ }^{1} \mathrm{~A}$ Sharif, ${ }^{2} \mathrm{P}$ Shorvon. ${ }^{1}$ Gastroenterology Centre, NW London Hospitals NHS Trust, London, UK; ${ }^{2}$ Department of Radiology, Central Middlesex Hospital, NW London Hospitals NHS Trust, London, UK

Introduction Acoustic Radiation Force Impulse (ARFI, Virtual Touch $^{\odot}$ ) elastography is a novel validated technique for measuring liver stiffness (LS), with advantages over transient elastography including greater accuracy in ascites or obesity. However, elastography has not been well studied in acutely ill patients with decompensated chronic liver disease (CLD). We report our experience in a consecutive controlled cohort in a secondary care setting. Aims: (1) To establish whether LS is significantly different in patients hospitalised for decompensated CLD from outpatient controls with proven cirrhosis; (2) To investigate correlation between ARFI and severity scores such as DF, GAH, Lille, Child Pugh and MELD

Methods ARFI was performed by a single radiologist, using a standard 10 observation technique. 108 patients were studied: (1) 60 hospitalised patients (13 AAH-acute alcoholic hepatitis with Bili >80; 19 DALD-decompensated ALD; 12 DCLD-decompensated CLD; 10 ALC-alcoholics without severe liver disease; 6 acute hepatitis), representing $39 \%$ of 152 consecutive cases seen by the liver service; and (2) 48 age and sex matched CLD controls (all never hospitalised, 33 with biopsy-proven advanced fibrosis/cirrhosis; 15 with clear clinical/radiological/endoscopic evidence of advanced CLD).

Results Validation: technical ARFI failure $<5 \%$, IOR/median $<0.5$ in $93 \%$. Compared with CLD controls, significantly higher mean shear velocity (SV) was seen in both (a) all 32 decompensated ALD patients (AAH+ALD-2.9 \pm 0.8 vs $2.4 \pm 0.8 \mathrm{~m} / \mathrm{s}, 99 \%$ CI 0.2 to 1.0 , $\mathrm{p}=0.001$ ) and (b) all 44 decompensated CLD patients (AAH+DALD $+\mathrm{DCLD}-2.8 \pm 0.8$ vs $2.4 \pm 0.8 \mathrm{~m} / \mathrm{s}, 99 \%$ CI 0.0 to $0.8, \mathrm{p}=0.006)$. In hospitalised patients with ALD (AAH+DALD+ALC) significant correlations were seen between mean SV and both DF $(r=0.55$, $\mathrm{p}<0.001)$ and GAH $(\mathrm{r}=0.38, \mathrm{p}=0.01)$, but not with Lille score Strong correlations were shown in all inpatients between SV and Child-Pugh score $(r=0.52, p<0.001)$, and also with MELD score $(\mathrm{r}=0.42, \mathrm{p}=0.002)$, but not in controls.

Conclusion In this "real world" study, ARFI elastography is an accurate and highly reproducible tool in assessing severity and prognosis in acutely ill patients with decompensated CLD, as shown by (a) increased LS in hospitalised ALD/CLD patients compared with cirrhotic controls, and importantly (b) further increases in LS reflect severity and adverse prognosis as shown by standard scores. As higher LS scores appear to reflect disease processes beyond fibrosis, the usefulness of this "real" measurement as an alternative to current "surrogate" prognostic markers merits further analysis in larger studies.

Competing interests None declared.

\section{PTU-025 A NEW PARADIGM IN THE NON-INVASIVE ASSESSMENT OF PORTAL HYPERTENSION: A SINGLE ULTRASOUND PROCEDURE INCORPORATING ACOUSTIC RADIATION FORCE IMPULSE (ARFI) ELASTOGRAPHY PREDICTS OVER 90\% OF OESOPHAGO-GASTRIC VARICES AND PORTAL GASTROPATHY}

doi:10.1136/gutjnl-2012-302514c.25

${ }^{1} \mathrm{D}$ । Sherman, ${ }^{*}{ }^{1} \mathrm{~K}$ Kandiah, ${ }^{*}{ }^{2} \mathrm{M}$ Jagtiani, ${ }^{1} \mathrm{~A}$ Sharif, ${ }^{2} \mathrm{P}$ Bassett, ${ }^{2} \mathrm{P}$ Shorvon. ${ }^{1}$ Gastroenterology Centre, NW London Hospitals NHS Trust, London, UK; ${ }^{2}$ Department of Radiology, Central Middlesex Hospital, NW London Hospitals NHS Trust, London, UK

Introduction Accurate non-invasive techniques for imaging portal hypertension (PHT) have long been the goal of hepatologists. However the sensitivity of standard ultrasound (US) alone is too low to achieve this. The combined use of elastography with US to detect PHT has not been investigated. ARFI (Virtual Touch ${ }^{\circledR}$ ) elastography is a novel validated technique for measuring liver stiffness (LS), with practical advantages over transient elastography including real-time scanning. We report our experience of this technique in assessing PHT in a consecutive secondary care cohort. Aims To establish whether the addition of LS estimation by ARFI to conventional US Doppler assessment of the spleno-portal venous system can reliably predict the presence of significant endoscopic PHT.

Methods Conventional US and elastography were performed simultaneously at one session by a single radiologist, using a Siemens Acuson $\mathrm{S} 2000^{\mathrm{TM}}$ and $4 \mathrm{C} 1$ probe $4 \mathrm{MHz}$ transducer and a 